\title{
Deletion of OSBPL2 in auditory cells increases cholesterol biosynthesis and drives reactive oxygen species production by inhibiting AMPK activity
}

Hongshun Wang ${ }^{1}$, Changsong Lin ${ }^{1}$, Jun Yao ${ }^{1,2}$, Hairong Shi ${ }^{1}$, Cui Zhang ${ }^{1}$, Qinjun Wei ${ }^{1,2,3}$, Yajie Lu ${ }^{1,2}$, Zhibin Chen ${ }^{4}$, Guangqian Xing ${ }^{4}$ and Xin Cao (1) ${ }^{1,2,3}$

\begin{abstract}
Oxysterol-binding protein like 2 (OSBPL2) was identified as a novel causal gene for autosomal dominant nonsyndromic hearing loss. However, the pathogenesis of OSBPL2 deficits in ADNSHL was still unclear. The function of OSBPL2 as a lipid-sensing regulator in multiple cellular processes suggested that OSBPL2 might play an important role in the regulation of cholesterol-homeostasis, which was essential for inner ear. In this study the potential roles of OSBPL2 in cholesterol biosynthesis and ROS production were investigated in Osbp/2-KO OC1 cells and osbp/2b-KO zebrafish. RNAseq-based analysis suggested that OSBPL2 was implicated in cholesterol biosynthesis and AMPK signaling pathway. Furthermore, Osbp/2/osbp/2b-KO resulted in a reduction of AMPK activity and up-regulation of Srebp2/srebp2, Hmgcr/ hmgcr and Hmgcs1/hmgcs1, key genes in the sterol biosynthetic pathway and associated with AMPK signaling. In addition, OSBPL2 was also found to interact with ATIC, key activator of AMPK. The levels of total cholesterol and ROS in OC1 cells or zebrafish inner ear were both increased in Osbp/2/osbp/2b-KO mutants and the mitochondrial damage was detected in Osbp/2-KO OC1 cells. This study uncovered the regulatory roles of OSBPL2 in cellular cholesterol biosynthesis and ROS production. These founds might contribute to the deep understanding of the pathogenesis of OSBPL2 mutation in ADNSHL.
\end{abstract}

\section{Introduction}

Cholesterol is an essential constituent of organelle membranes in eukaryotic cells, where it is implicated in various cellular processes, including the regulation of membrane permeability, membrane trafficking, signal transduction, and endocytosis ${ }^{1-5}$. The intracellular cholesterol synthesis, efflux (egress), and uptake (ingress) are tightly controlled by the feedback on direct interactions

\footnotetext{
Correspondence: Xin Cao (caoxin@njmu.edu.cn)

${ }^{1}$ Department of Medical Genetics, School of Basic Medical Science, Nanjing Medical University, Nanjing, China

${ }^{2}$ Jiangsu Key Laboratory of Xenotransplantation, Nanjing Medical University, Nanjing, China

Full list of author information is available at the end of the article.

These authors contributed equally: Hongshun Wang, Changsong Lin, Jun Yao Edited by A. Verkhratsky
}

of cholesterol or oxysterols with regulatory factors, such as 3-hydroxy-3-methylglutaryl-coenzyme A reductase (HMGCR), sterol responsive element-binding protein (SREBP) cleavage activating protein (SCAP), acylcoenzyme A: cholesterol acyltransferase (ACAT) and liver X receptors (LXRs). These factors act as sterol sensors and effectively regulate cholesterol-homeostasis by a serial of post-transcriptional and post-translational mechanisms ${ }^{6-9}$. Increasing evidences suggest that deregulation of cholesterol-homeostasis is the causative or characteristic factor of numerous pathologies, including cardiovascular disease, cerebrovascular disease, metabolic diseases, tumourigenesis and neurodegenerative diseases ${ }^{10-13}$. It was also noteworthy that sensorineural hearing loss (SNHL) is observed as one of the 
characteristic features in atherosclerosis and some genetic syndromes that affect intracellular cholesterol synthesis or transport ${ }^{14,15}$.

Oxysterol-binding protein like 2 (OSBPL2, OMIM: 606731), a member of the oxysterol-binding proteins (OSBP) related proteins (ORPs) family, is known as a sterol sensor and transporter that modulates lipid/cholesterol metabolism, steroid hormone synthesis, cell signaling, vesicular trafficking and cytoskeleton formation ${ }^{16-20}$. Our previous work described the association of human mutations in OSBPL2 with autosomal dominant non-syndromic hearing loss (ADNSHL) in a large affected Chinese family ${ }^{21}$, which was also reported in an affected German family by Thoenes et $\mathrm{al}^{22}$. The pathogenesis of OSBPL2 deficits in ADNSHL was still unclear, however, the importance of cholesterol-homeostasis in inner ear as well as the unique sensitivity of the auditory organ to changes in cholesterol localization ${ }^{23,24}$ suggested that the potential role of OSBPL2 in lipid/cholesterol-homeostasis was essential to the auditory function. In fact, the cellular function of OSBPL2 has been reported to be associated with cholesterol and triacylglycerol homeostasis in distinct cell lines ${ }^{25,26}$. Escajadillo et al. found that reduced amounts of multiple steroid metabolites were observed in OSBPL2knockdown H295R adrenocortical cells, and OSBPL2silencing led to the suppression of key factors for cortisol production, resulting in increased cellular cholesterol and reduced 22(R)-hydroxycholesterol [22-(R)-OHC] and 7ketocholesterol $(7-\mathrm{KC})^{16}$. It was suggested that the OSBPL2 deficits might result in the loss of tightly-regulated cholesterol-homeostasis response in inner ear and lead to the auditory dysfunction. Considering the ubiquitous expression of OSBPL2 in diverse cell types and multiple cellular functions of this sterol sensor, the potential pathogenesis of OSBPL2 deficits in ADNSHL still needed to be further investigated in the functional or dysfunctional context of specific cell types in vitro and in vivo.

In this study, $\mathrm{OC} 1$ auditory cell and zebrafish as well as their Osbpl2/osbpl2b-knockout (KO) mutants were used to investigate the potential role of OSBPL2 in the regulation of cholesterol-homeostasis. Our findings showed that Osbpl2/ osbpl2b-KO led to excessive cholesterol biosynthesis by inhibiting AMPK signaling pathway and induced the production of intracellular reactive oxygen species (ROS), which might be the causative factor of mitochondrial damage in Osbpl2-KO OC1 cells. This work would contribute to deep understanding of the molecular function of OSBPL2 in auditory cells/inner ear, and provide an insight of the pathogenesis of OSBPL2-deficit implicated in ADNSHL.

\section{Results}

\section{Osbp/2-KO interferes with AMPK signaling}

RNA-seq was performed to identify genes and pathways affected by OSBPL2-function/dysfunction. The expression profiles were examined by using multidimensional scaling (MDS) analysis to visualize the profiles and by comparing differentially expressed genes (DEGs). MDS analysis showed that significant transcriptional changes were detected in Osbpl2-KO OC1 cells, with 1435 (11.66\%) DEGs out of 12309 detectable genes (Fig. 1a and Fig. S1). $\mathrm{GO}$ analysis of this gene set revealed that the enriched clusters were related to the cholesterol biosynthesis, lipid transport and sterol metabolic process (Fig. 1b). Then, we analysed DEGs associated with cholesterol biosynthesis by KEGG pathway analysis. The results showed that AMPK signaling was predicted to be the most significant pathway (Fig. 1c). To validate this, we measured the expression of top-ranked genes in each cluster and additional genes involved in AMPK signaling pathway and performed hierarchical Euclidean distance analysis (Fig. 1d). In Osbpl2-KO cells, the majority of genes related to cholesterol biosynthesis were upregulated and clustered. These results suggested that Osbpl 2 was involved in the regulation of cholesterol biosynthesis and AMPK signaling pathway.

\section{Osbp/2/osbp/2b -KO inhibits AMPK activity and increases cholesterol synthesis}

To investigate the effect of Osbpl2-KO on AMPK signaling pathway and cholesterol synthesis, the protein levels of phosphorylated AMPK-Thr172 and total AMPK were measured in Osbpl2-KO/WT cells. Considering that osbpl $2 b$ is an orthologous gene of human OSBPL $2^{27}$, osbpl2b-KO zebrafish was also constructed (Fig. S2) and used to investigate AMPK activity in inner ear. Meanwhile, AMPK activation was also assessed by examining phosphorylated ACC-Ser79, which was a direct target of activated AMPK and commonly used as an indicator of AMPK activity in cells. The results showed that phosphorylated AMPK-Thr172 and phosphorylated ACCSer79 were both detected at lower levels in Osbpl2-KO cell (Fig. 2a). A similar effect was observed in osbpl2b-KO zebrafish inner ear (Fig. 2b). The protein levels of Srebp2, Hmgcr and Hmgcs1, key regulators in AMPK signaling pathway and cholesterol biosynthesis, were also measured in Osbpl2/osbpl2b-KO mutants. The expression of Srebp2/srebp2, Hmgcr/hmgcr and Hmgcs1/hmgcs1 in mRNA and protein levels were increased both in Osbpl2$\mathrm{KO}$ cells (Fig. 2c, Fig. S3a) and osbpl2b-KO zebrafish inner ear (Fig. 2d, Fig. S3b). Besides, the cleavage processing of Srebp $2 / \operatorname{srebp} 2$, as reflected by the amounts of the precursor $(126 \mathrm{kDa})$ and nuclear active forms $(55 \mathrm{kDa})$ were determined. The expression of nuclear Srebp $2 /$ srebp 2 were markedly increased both in Osbpl2-KO cells (Fig. 2c) and osbpl2b-KO zebrafish inner ear (Fig. 2d). In addition, as expected, significant increases of TC content were observed in Osbpl2-KO cells (Fig. 2e) and osbpl2b-KO zebrafish inner ear (Fig. 2f). The above results indicated 
A

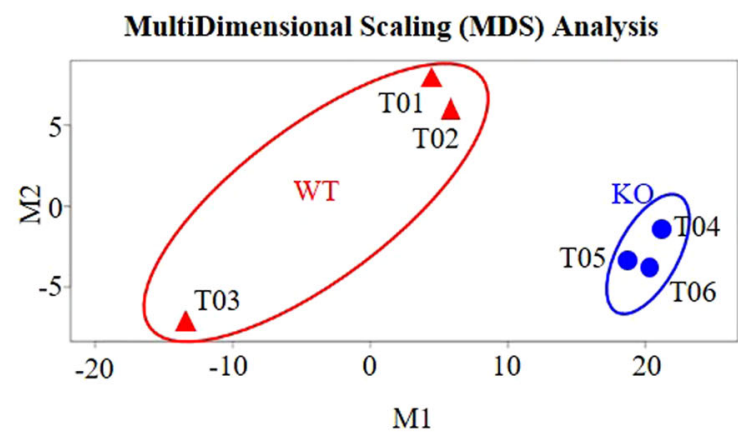

B

\begin{tabular}{lc}
\hline GO Term: Biological Process & $\boldsymbol{P}$-Value \\
\hline Response to glucocorticoid & $1.48 \mathrm{E}-03$ \\
\hline Cholesterol biosynthesis process & $1.63 \mathrm{E}-03$ \\
Lipid Transport & $2.89 \mathrm{E}-02$ \\
Fatty acid biosynthetic process & $5.26 \mathrm{E}-02$ \\
Response to glucose & $6.26 \mathrm{E}-02$ \\
\hline
\end{tabular}

$\mathrm{C}$

The Proportions of DEGs Enriched in the Signaling Pathways

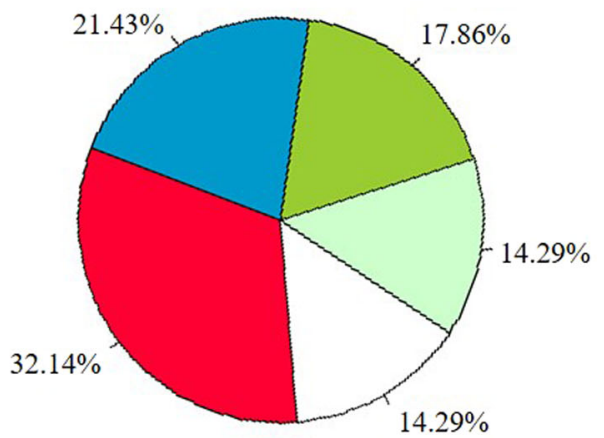

AMPK signaling pathway $\square$ TNF signaling pathway $\square$ PPAR signaling pathway $\square$ FoxO signaling pathway $\square$ JAK-STAT signaling pathway

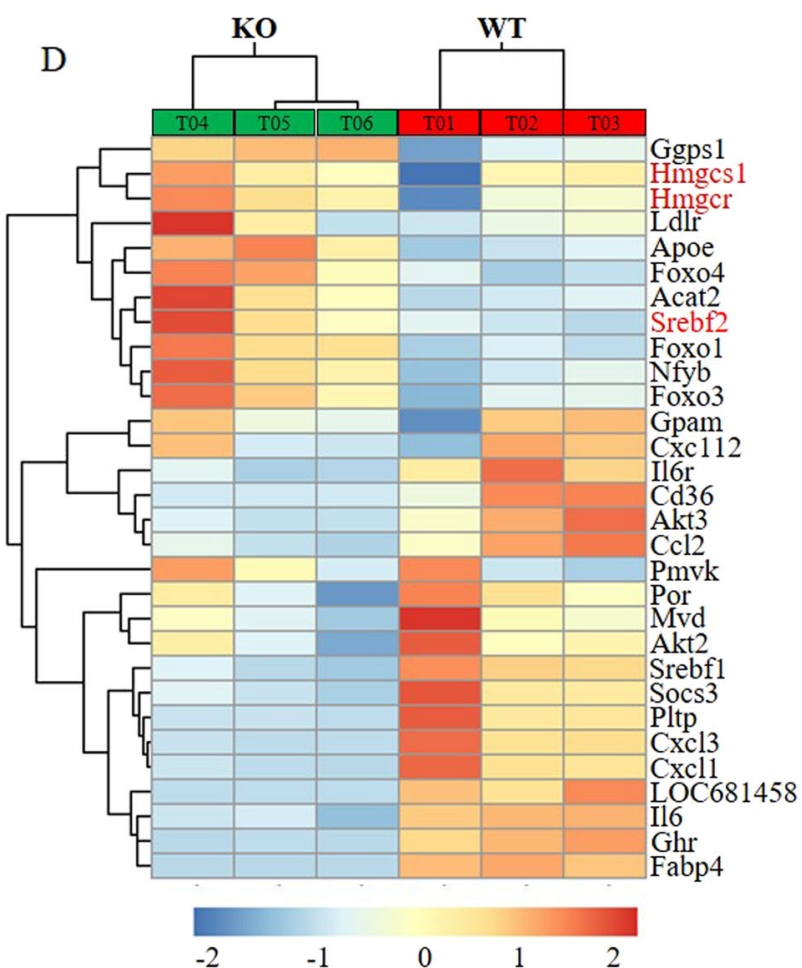

Fig. 1 RNA-seq of Osbp/2-KO and wild-type (WT) OC1 cells. a Multi-Dimensional scaling (MDS) analysis of RNA-seq profiles collected from Osbp/2$\mathrm{KO}$ and WT OC1 cells $(n=3)$. b The enrichment of biological processes was determined by gene ontology (GO) analysis. P-value was assessed by Benjamini procedure. $\mathbf{c}$ The gene enrichment was determined by KEGG pathway analysis, the \% scale represented the proportions of DEGs enriched in the signaling pathways to total DEGs associated with cholesterol biosynthesis. $\mathbf{d}$ Genes enriched in AMPK signaling pathway were shown in the heat map. Data was normalized by logarithm of 2

that Osbpl2/osbpl2b-KO led to the inhibition of AMPK activity and aberrant cholesterol biosynthesis/accumulation in auditory $\mathrm{OC} 1$ cells and zebrafish inner ear.

\section{Osbp/2-KO increases cholesterol biosynthesis by suppressing AMPK signaling pathway}

Inhibition of AMPK was conducted in $\mathrm{OC} 1$ cells to validate its effect on cholesterol biosynthesis. OC1 cells were treated with $5 \mathrm{nM}$ Compound $C$, an inhibitor of AMPK, and the levels of phosphorylated AMPK-Thr172, Srebp2, Hmgcr, and Hmgcs 1 were determined by Western blot analysis. The results showed that the inhibition of AMPK led to a decrease of phosphorylated AMPKThr172, but resulted in the up-regulation of nuclear Srebp2, Hmgcr, and Hmgcs1 (Fig. 3a). Meanwhile, a significant increase of intracellular TC content was detected by the treatment with Compound $\mathrm{C}$ (Fig. 3b). On the other hand, we wanted to know whether cholesterol synthesis returned to normal levels after AMPK activity inhibition was rescued in Osbpl2-KO OC1 cells. The results showed that after AMPK re-activation, the expression of phosphorylated AMPK-Thr172, nuclear 

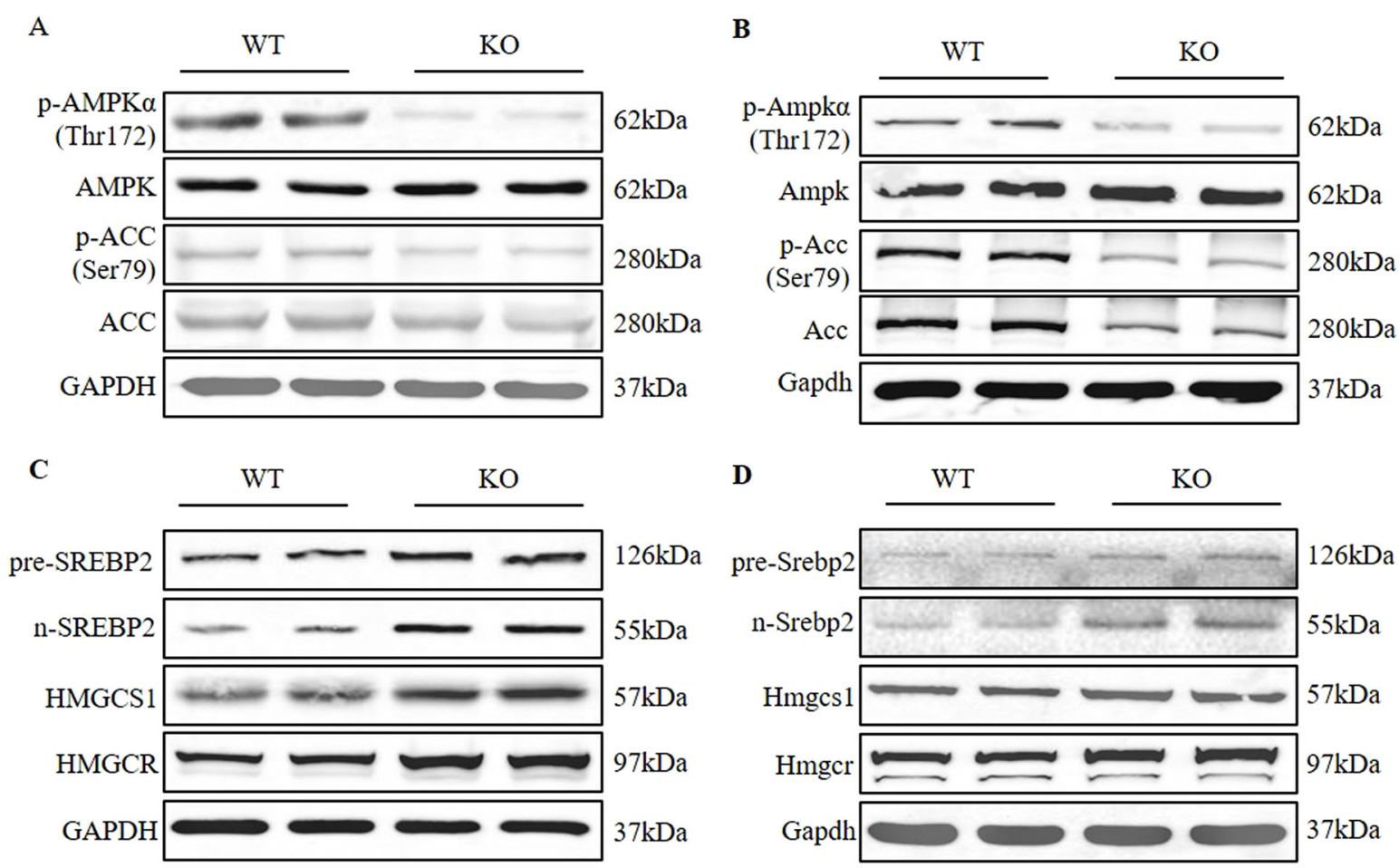

$\mathbf{E}$

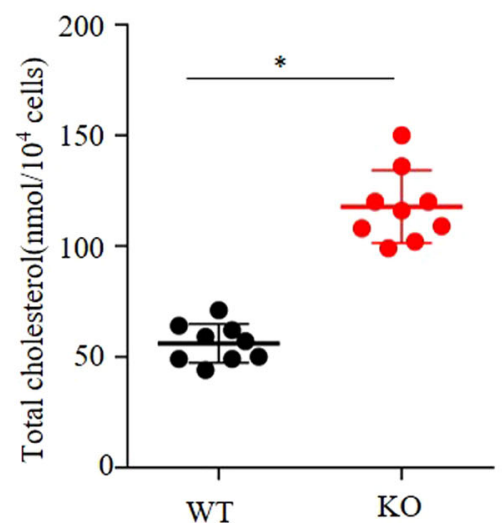

$\mathbf{F}$

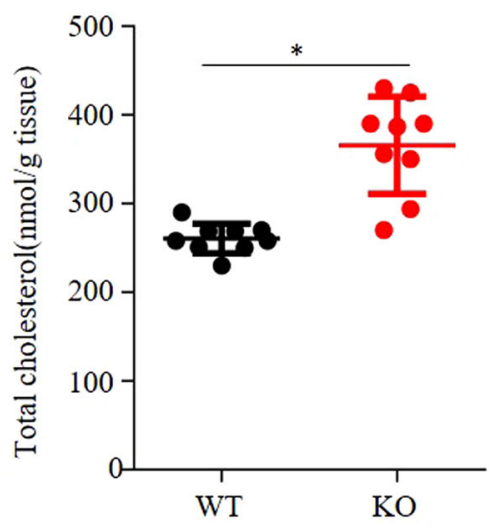

Fig. 2 Osbpl2/osbp/2b-KO inhibited AMPK activity and increased intracellular cholesterol levels. a Western blot analysis of AMPK (total and Thr172), ACC (total and Ser79) and GAPDH in Osbp/2-KO/WT OC1 cells. b Western blot analysis of Ampk (total and Thr172), Acc (total and Ser79) and Gapdh in osbp/2b-KO/WT zebrafish inner ear. c Western blot analysis of pre-SREBP2, n-SREBP2, HMGCR, HMGCS1 and GAPDH in Osbp/2-KO/WT OC1 cells. d Western blot analysis of pre-Srebp2, n-Srebp2, Hmgcr, Hmgcs1, and Gapdh in osbpl2b-KO/WT zebrafish inner ear. e Total cholesterol levels in Osbp/2-KO/WT OC1 cells $(n=9)$, * $p<0.05$. f Total cholesterol levels in Osbpl2-KO/WT zebrafish inner ear $(n=9)$, * $p<0.05$

Srebp2, Hmgcs1 and Hmgcr showed a tendency to return normal, and cholesterol content also recovered (Fig. 3a, b). These results suggested that AMPK might play an important role in cholesterol biosynthesis.

To further confirm the effect of AMPK on cholesterol biosynthesis, AMPK siRNA was used to silence the expression of AMPK. Silencing AMPK up-regulated the expression of nuclear Srebp2, Hmgcr, and Hmgcs1 (Fig. 3c), leading to increased cellular TC levels (Fig. 3d). AMPK overexpression had little effect on the expression of nuclear Srebp2, Hmgcr, and Hmgcs1 (Fig. 3e) as well as cholesterol biosynthesis (Fig. 3f). These results indicated that the biosynthesis of cholesterol was related to the activity of AMPK but independent of the AMPK expression levels. Therefore, it was suggested that OSBPL2 plays an important role in the regulation on cholesterol biosynthesis through AMPK signaling pathway, and OSBPL2 deficit induced aberrant cholesterol biosynthesis/accumulation by inhibiting AMPK activity. 


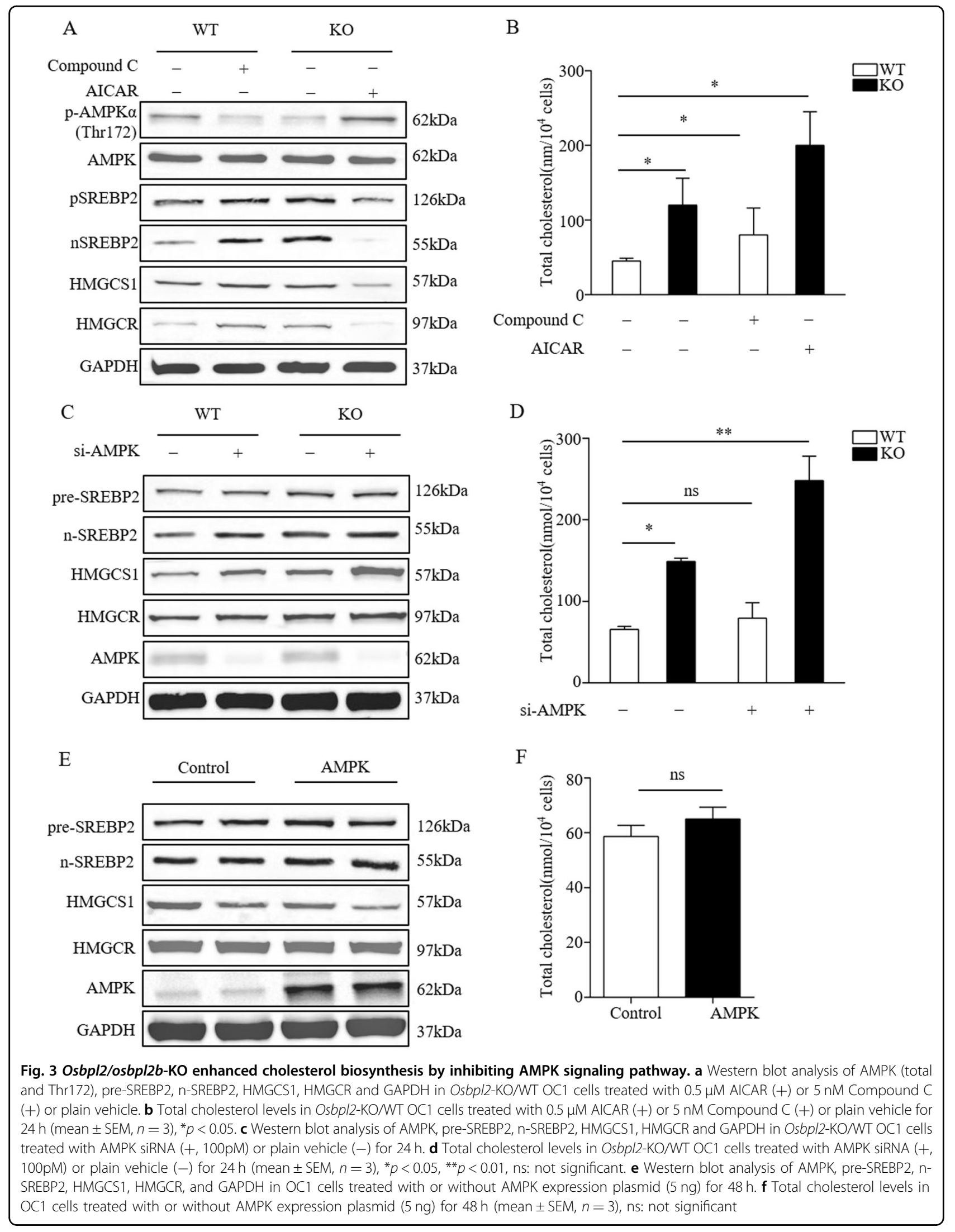




\section{OSBPL2 interacts with ATIC to regulate AMPK activity}

The regulatory mechanisms of OSBPL2 on AMPK activity were further investigated by proteomic analysis of the OSBPL2 interactome. Flag-tagged OSBPL2 was expressed in HEK293Ta cells, followed by a pulldown of complexes with anti-Flag magnetic beads. Mass spectrometric analysis identified 47 proteins that were found specifically in the Flag-OSBPL2 complexes (Table S1). Among these binding partners of OSBPL2, ATIC, a bifunctional purine biosynthesis protein, is known to be the key AMPK effector. The interaction of OSBPL2 with ATIC was validated by Co-IP. Abundant HA-ATIC was detected in Flag-OSBPL2 complexes, but absent in the negative control with plain Flag (Fig. 4a), supporting a specific interaction of ATIC with OSBPL2. Similarly, GST pulldown assay showed that GST-OSBPL2 interacted with His-ATIC, but no interactions were detected in the negative control with plain GST (Fig. 4b). The truncated ATIC was used to determine the binding site of ATIC interacting with OSBPL2 (Fig. 4c). The OSBPL2-ATIC interaction remained when ATIC lacked methylglyoxal synthase-like (MGS) domain and carboxyl terminal (CT) domain, but not detected at the absence of 5aminoimidazole-4-carboxamide ribonucleotide formyltransferase and inosine monophosphate (IMP) cyclohydrolase (AICARFT_IMPCHase) domain (Fig. 4a). These results indicated that OSBPL2 interacted with ATIC and the AICARFT_IMPCHase domain of ATIC was essential for OSBPL2-ATIC interaction.

ATIC is a rate-limiting enzyme and affects intracellular levels of AICAR, which behaves as an AMP analog that allosterically activates AMPK. ATIC is also capable of affecting AMPK activity by regulating the ninth step of de novo purine biosynthesis ${ }^{28,29}$. It was noteworthy that the AICAR level was decreased in Osbpl2-KO cells (Fig. 4d). In addition, the level of phosphorylated AMPK-Thr172 decreased in ATIC-overexpressing OC1 cells (Fig. 4e), but increased in ATIC-siRNA knockdown OC1 cells (Fig. 4f). To determine the effect of ATIC on AMPK activity, the AICAR levels were measured in the case of ATIC overexpression or knockdown. The results showed that AICAR was decreased in ATIC-overexpressed OC1 cells (Fig. S4a), while increased in ATIC-siRNA-knockdown OC1 cells (Fig. S4b). These results suggested that the OSBPL2 deficit led to the destruction of the OSBPL2ATIC interaction, resulting in decreased levels of AICAR and inhibition of AMPK activity.

\section{Intracellular cholesterol accumulation induces ROS and cause mitochondrial damage}

Considering the effect of excessive cholesterol on intracellular ROS production, the increased TC content induced by Osbpl2/osbpl $2 b$-KO might also be of sufficient magnitude to affect ROS levels. As was expected, the basal
ROS levels of Osbpl2-KO OC1 cells were 50\% higher than that of WT controls (Fig. 5a). Similarly, osbpl $2 b-\mathrm{KO}$ increased the ROS levels by $30 \%$ in zebrafish inner ear (Fig. 5b). A significant increase of ROS levels was also detected in Osbpl2-KO OC1 cells treated with different concentrations of cholesterol (Fig. 5c). However, the ROS levels in Osbpl2-KO OC1 cells treated with $\mathrm{M} \beta \mathrm{CD}$ showed a tendency to return normal. (Fig. 5d). The effect of ROS on the subcellular features was assessed in Osbpl2-KO cells. Compared with WT controls, swollen mitochondria with missing cristae were observed in Osbpl2-KO cells (Fig. 5e). In addition, the mitochondrial membrane potential was also detected decreased in Osbpl2-KO OC1 cells (Fig. 5f). Meanwhile, several antioxidant genes, including Sod1, Sod2, and Cat, were significantly downregulated in Osbpl2-KO OC1 cells (Fig. S5). These results strongly suggested the mitochondria damage in Osbpl2-KO OC1 cells.

The above results suggested that the OSBPL2-ATIC interaction contributed to intracellular AICAR accumulation in WT cells, which activated AMPK signaling pathway and controlled the nuclear transfer of SREBP2 to bind sterol response element (SRE) $)^{30,31}$, resulting in regular cholesterol biosynthesis. In addition, AMPK regulated cholesterol synthesis by directly controlling the activity of HMGCR ${ }^{32}$. On the other hand, OSBPL2 deficit interfered the OSBPL2-ATIC interaction and reduced the intracellular AICAR level as well as AMPK activity, which enhanced nuclear transfer of SREBP2 and upregulated the expression HMGCR and HMGCS1, resulting in excessive cholesterol biosynthesis and ROS accumulation (Fig. 6).

\section{Discussion}

Since OSBPL2 was identified as the ADNSHL-causative gene $^{21,22}$, it has drawn much attention to the molecular function of OSBPL2 implicated in multiple biological processes. The current studies revealed the role of OSBPL2 in lipid/cholesterol transport and metabolism as well as cell signaling in diverse cell types, which suggest that OSBPL2 might play an important role in the regulation of intracellular cholesterol-homeostasis. To gain a deep understanding of the physiologic function of OSBPL2 in inner ear, we employed Osbpl2-KO/knockdown OC1 auditory cells and osbpl2b-KO zebrafish to investigate the regulatory mechanism of OSBPL2 in cholesterol-homeostasis. The RNA-seq analysis revealed the alteration of Osbpl2-KO OC1 cells in the biological process categories: lipid transport, lipid metabolic process, lipid storage and cholesterol transport, corresponding to estradiol and cholesterol biosynthesis. KEGG Pathway analysis showed that AMPK signaling pathway was the most significant pathway. Osbpl2-KO resulted in up-regulation of Srebp2, Hmgcr and Hmgcs1, key genes in the sterol biosynthetic pathway and associated with 


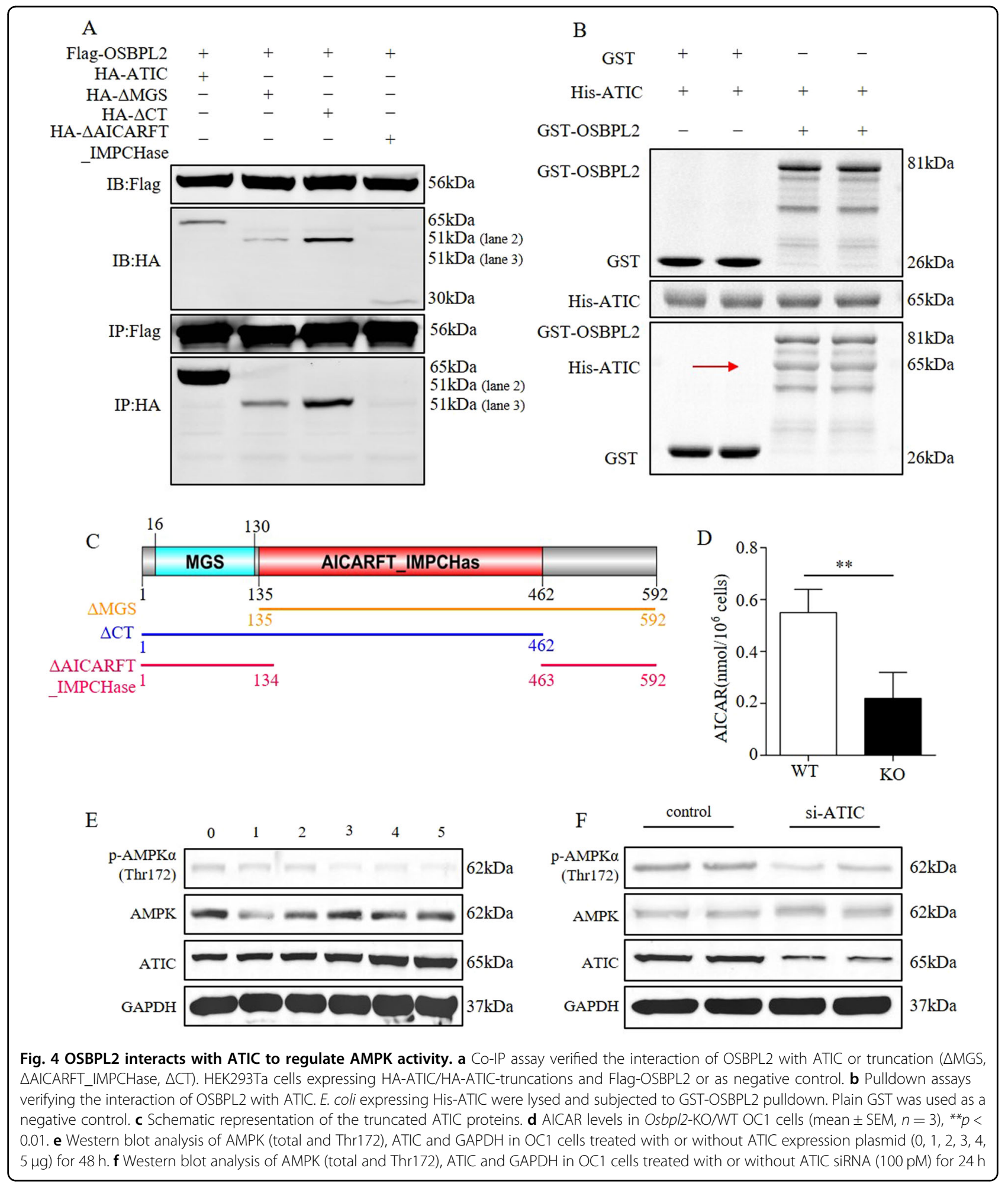

AMPK signaling. These results strongly suggested that Osbpl2/osbpl2b deficits inhibited AMPK activity and enhanced cholesterol biosynthesis.

AMPK is the downstream component of a kinase cascade that acts as a gauge of cellular energy levels.
Recently, accumulating evidence has demonstrated that AMPK is also the key regulator of metabolism, protein synthesis, cell growth and apoptosis, cell polarity and ion flux $^{33-35}$. There was a strong correlation between low AMPK activation status with insulin resistance, obesity 

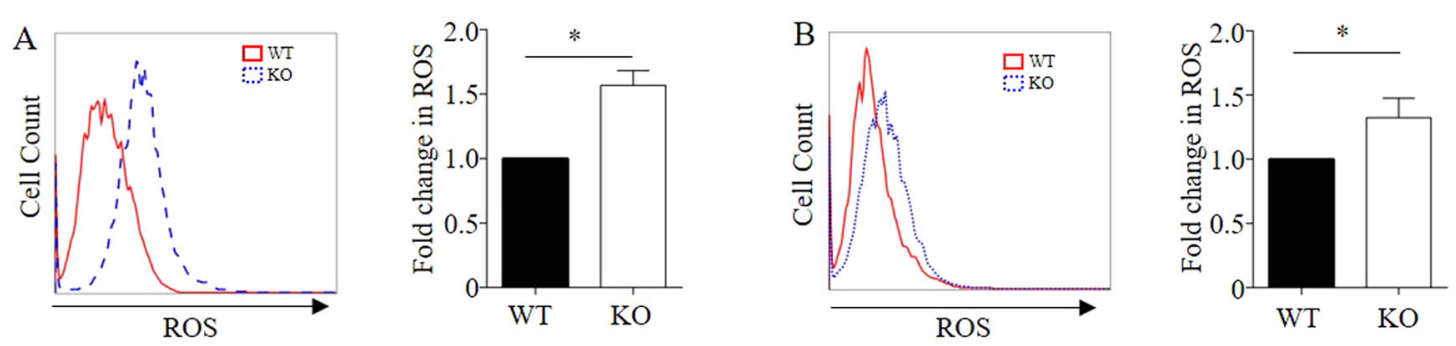

C
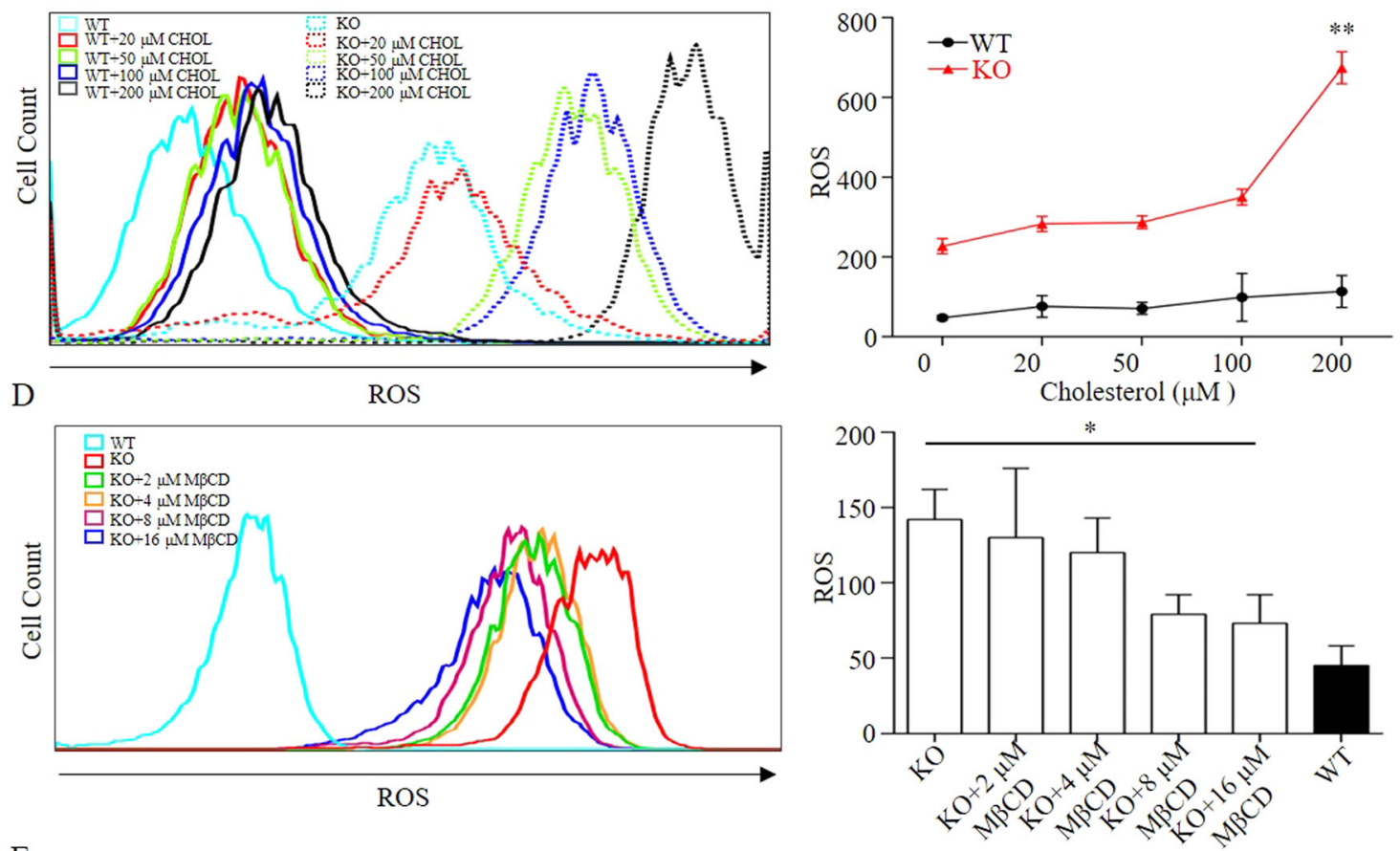

E
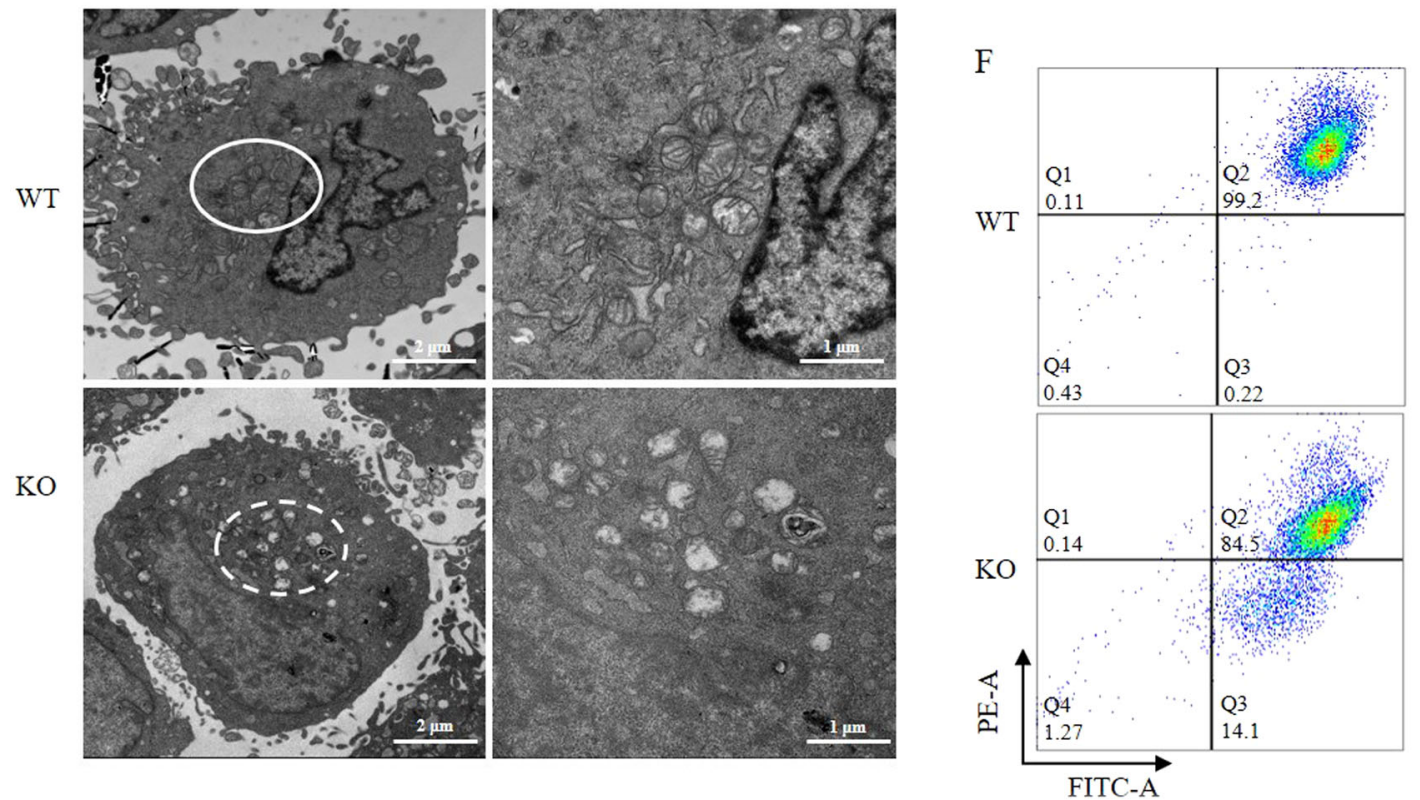

Fig. 5 (See legend on next page.) 
(see figure on previous page)

Fig. 5 Intracellular cholesterol accumulation induces ROS and causes mitochondrial damage. a Mitochondrial ROS levels in Osbp/2-KO/WT OC1 cells. Left: representative histogram for CellROX ${ }^{\mathrm{TM}}$ Deep Red staining. Right: relative fold changes of ROS (mean \pm SEM, $n=3$ ), ${ }^{*} p<0.05$. $\mathbf{b}$ Mitochondrial ROS levels in osbp/2b-KO/WT zebrafish inner ear. Left: representative histogram for CellROX ${ }^{\mathrm{TM}}$ Deep Red staining. Right: relative fold changes of ROS (mean \pm SEM, $n=3$ ), ${ }^{*} p<0.05$. c Mitochondrial ROS levels in Osbpl2-KONT OC1 cells treated with different concentrations of cholesterol (0, 20, 50, 100, $200 \mu \mathrm{M})$ for $12 \mathrm{~h}$. Left: representative histogram for CellROX'M Deep Red staining. Right: relative fold changes of ROS (mean $\pm \mathrm{SEM}, n=3),{ }^{* *} p<0.01$. d Mitochondrial ROS levels in Osbp/2-KO/WT OC1 cells treated with different concentrations of M $B C D(0,2,4,8,16 \mathrm{nM})$ for $12 \mathrm{~h}$. Left: representative histogram for CellROX ${ }^{\mathrm{TM}}$ Deep Red staining. Right: relative fold changes of ROS (mean $\pm \operatorname{SEM}, n=3$ ), ${ }^{*} p<0.05$. e The cellular ultrastructure as analyzed by transmission electron microscopy (TEM) in Osbp/2-KO/WT OC1 cells. $\mathbf{f}$ The membrane potential levels of Osbp/2-KO/WT

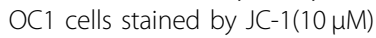

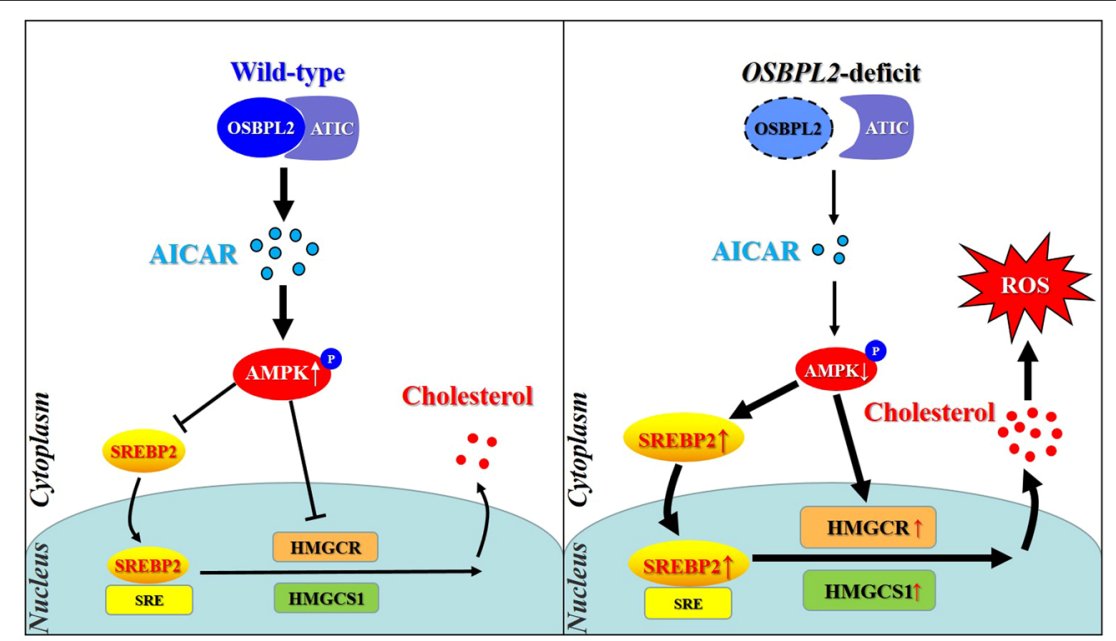

Fig. 6 Proposed regulatory mechanism of OSBPL2 on intracellular cholesterol biosynthesis and ROS production. SRE: sterol response element. ROS: reactive oxygen species

and sedentary lifestyle ${ }^{36-38}$. Decreased AMPK activation is implicated in human metabolic disorders associated with increased cancer risk $^{39}$. The previous studies had revealed that the AMPK signaling pathway was closely related to hearing loss. LKB1, upstream kinase of AMPK, is expressed from the cuticular plate to the nuclei of hair cells and contributes to maintaining the development and structural stability of cochlear hair cells and stereocilia bundles $^{40}$. Hill et al. found that long-term phosphorylation of AMPK caused by noise exposure could inversely lead to a decreased auditory function along with the loss of hair cell and synaptic ribbon ${ }^{41}$. In fact, AMPK controls cellular lipid metabolism through direct phosphorylation of ACC and ACC1. AMPK also phosphorylates and inhibits SREBP1/2 and HMGCR, which leads to a preprogramming of lipid and intracellular sterol biosynthesis $^{32,42}$. In the present work, AMPK inhibition increased intracellular cholesterol synthesis, which was observed both in Osbpl2/osbpl2b-KO mutants. It was suggested that inhibition of AMPK promotes nuclear transfer of SREBP2, which promotes the expression of Hmgcs1 and Himgcr.
The interaction of OSBPL2 with ATIC, the key effector of AMPK activity, further confirmed the regulatory role of OSBPL2 on AMPK activity. We found that OSBPL2 combined with the AICARFT_IMPCHas domain of ATIC and OSBPL2-deficient resulted in the inhibition of AMPK activity. ATIC is a cytosolic enzyme $(64 \mathrm{kDa})$ containing a transformylase domain (residues 200-593), which transfers a formyl group to the AMP analog AICAR to produce the intermediate formyl-AICAR (FAICAR) and IMP ${ }^{43,44}$. AICAR is a nucleoside that is converted inside the cells to its monophosphorylated form, 5-amino-4-imidazolecarboxamide ribotide (ZMP) by adenylate kinase. Thus, AICAR behaves as an AMP analog that allosterically activates AMPK. Recent studies demonstrated that inhibition of ATIC led to a subsequent rise in intracellular ZMP and resulted in the increased AMPK activity ${ }^{45}$. Furthermore, Li et al. found that ATIC overexpression inhibited AMPK activity, and homodimerization of ATIC was essential for the AICAR transformylase (AICART) activity, as the active site of this enzyme was formed at the interface of two interacting ATIC monomers with each molecule contributing residues ${ }^{29}$. Daniel et al. found that an inhibitor of ATIC 
homodimerization up-regulated intracellular ZMP via a metabolic block on the ninth step of the de novo purine biosynthesis pathway ${ }^{28}$. In the present work, we found that OSBPL2 binds to the AICARF domain of ATIC and regulates the cellular levels of AICAR. The present data were consistent with a model in which OSBPL2 deficits led to the exposure of the AICARF domain, inhibited the cellular levels of AICAR, and suppressed AMPK activity.

Beyond the role of OSBPL2 in lipid/cholesterol metabolism and transport, the present study uncovered OSBPL2 as a key regulator in cellular cholesterolbiosynthesis using auditory $\mathrm{OC} 1$ cells and zebrafish models. Consistent with RNA-seq analysis, we found that the OSBPL2 deficits led to an inhibition of AMPK activity and aberrant cellular cholesterol biosynthesis, which suggested the deregulation of cholesterol homeostasis. Increasing evidences had suggested the importance of lipid/cholesterol-homeostasis in auditory function. Some genetic syndromes, such as Niemann-Pick type $\mathrm{C}$ and Smith-Lemli-Opitz, that affect cholesterol intracellular transport/synthesis display devastating neurological phenotypes including SNHL ${ }^{46,47}$. The epidemiology studies had suggested the potential association of hypercholesterolemia and SNHL. Interestingly, it was found that Apolipoprotein E (ApoE) knockout mice developed remarkable hyperlipedimia, atherosclerosis and hearing impairment, which might share the similar pathogenesis of OSBPL2 deficit in SNHL ${ }^{48}$. In our study, excessive cholesterol levels and increased ROS were both detected in Osbpl2-KO OC1 cells and osbpl2b-KO zebrafish inner ear. ROS are endogenous products of metabolism and essential as the second message in cellular signaling involved in multiple cellular process. However, excessive ROS accumulation can damage cellular proteins, lipids and DNA, leading to cell dysfunction, senescence and even cell death ${ }^{49-51}$. Gonzalo Alba et al reported that excessive cholesterol enhanced ROS production in human neutrophils ${ }^{52}$. In the hyperlipidemia/hyperlipedimia mice/ rats models, it was found that long-term high-fat diets led to the activation/elevation of NADPH Oxidase Catalytic Subunit-Like 3 (NOX3), uncoupling protein 2 (UCP2) and uncoupling protein 3 (UCP3), causing mitochondrial damage and apoptosis in inner ear ${ }^{53,54}$. Mitochondria are a major source of ROS, but easily damaged by ROS. This mitochondrial oxidative damage contributes to cellular dysfunction or cell death in various diseases ${ }^{55}$. Our study suggested that Osbpl2/osbpl2b-KO enhanced the ROS production and led to mitochondrial damage and membrane potential reduction in auditory OC1 cells, which might be implicated in the pathogenesis of OSBPL2 deficit in SNHL development.

In summary, the present work uncovered the regulatory mechanism of OSBPL2 on cholesterol-biosynthesis/ homeostasis in OC1 auditory cells and inner ear of zebrafish. It was suggested that OSBPL2 and other key regulators/effectors in AMPK signaling pathway were critical for cholesterol-homeostasis in inner ear and maintenance of hearing function. Our findings contributed to elucidating the pathogenesis/physiopathology of OSBPL2 mutation and the potential linkage of deregulation of cholesterol-homeostasis and SNHL.

\section{Materials and methods \\ Antibodies and reagents}

Antibodies for adenosine $5^{\prime}$-monophosphate (AMP)activated protein kinase (AMPK/Ampk) (\#2793), Flag-Tag (\#2368), His-Tag (\#12698), GST-Tag (\#2624), GAPDH (\#5174) and phosphospecific antibodies for AMPK/Ampk -pThr172 (\#2535) were purchased from Cell Signaling Technology (Danvers, MA, USA). Antibodies for HMGCR/Hmgcr (\#ab174830), 5-aminoimidazole-4carboxamide ribonucleotide formyltransferase/inosine monophosphate cyclohydrolase (ATIC) (\#ab188321), HATag (\#ab1424) and phosphospecific antibodies for acetylCoA carboxylase (ACC/Acc) pSer79 (\#ab68191) were purchased from Abcam (Cambridge, UK). Antibodies for ACC/Acc (\#21923-1-AP), 3-hydroxy-3-methylglutarylCoA synthase 1 (HMGCS1) (\#17643-1-AP) and sterol regulatory element-binding protein 2 SREBP2/Srebp2 (\#A13049) were from Proteintech (Chicago, IL, USA). Antibody for Gapdh (\#GTX100118) was purchased from GeneTex (Alton, CA, USA). IRDye $800 \mathrm{CW}$ goat antiMouse IgG (\#926-32213) and IRDye 680LT goat antiRabbit IgG (\#926-68021) were purchased from LI-COR Biosciences (Lincoln, Nebraska, USA). Cholesterol (\#C8667) and methyl- $\beta$-cyclodextrin (\#C4555) were purchased from Sigma-ldrich (St. Louis, MO, USA). Aminoimidazole carboxamide ribonucleotide (AICAR) (\#S1802) and Compound C (\#S7840) were purchased from Selleckchem (Houston, TX, USA).

\section{Animals}

Osbpl2b-KO and wild-type (WT) zebrafish were maintained using standard procedures and used in accordance with the protocols approved by the Institutional Animal Care and Use Committee (IACUC) of the Nanjing Medical University. The fish were kept at $28^{\circ} \mathrm{C}$ in buffered reverse osmosis water with a standard light/dark cycle of $14 \mathrm{~h} / 10 \mathrm{~h}$ and fed Aquatox Fish Diet flakes (Zeigler, China) twice per day, supplemented with brine shrimp.

\section{Cell culture and transfection}

Osbpl2b-KO/WT OC1 cells were grown in Dulbecco's Modified Eagle's Medium (DMEM, Gibco, USA) supplemented with $10 \%$ Foetal Bovine Serum (FBS, Gibco, USA) at $33{ }^{\circ} \mathrm{C}$ with $10 \% \mathrm{CO}_{2}$, subcultured every 2 day at a density of $1 / 4$ or every $3 \mathrm{~d}$ at a density of $1 / 8$ and discarded after passage 8. HEK293Ta cells were cultured in 
the above medium at $37^{\circ} \mathrm{C}$ with $5 \% \mathrm{CO}_{2}$. Plasmids were transfected into cells using Lipofectamine 3000 Reagent (Invitrogen, USA) when the cells achieved 70\%-90\% confluent. SiRNAs were transfected into cells using XtremeGENE siRNA Transfection Reagent (Roche, USA) at $30-50 \%$ of cell confluent.

\section{Quantitative real-time polymerase chain reaction}

Cells or tissues were homogenized in TRIzol Reagent (Life, USA) and total RNA were extracted according to the manufacturer's instructions. Complementary DNA (cDNA) was synthesized via HiScript II One Step RT-PCR Kit (Vazyme, China). The quantitative real-time polymerase chain reaction (qRT-PCR) was performed on a StepOne Plus system (Applied Biosystems, USA) using ChamQ SYBR qPCR Master Mix (Vazyme, China). The comparative CT method $\left(2^{-\Delta \Delta C T}\right)$ was used to analyze gene expression. GAPDH was used as an internal control. The primer sequences were listed in Supplemental Table S2.

\section{Western blot analysis}

Cells were washed three times with cold PBS and then lysed with RIPA Lysis Buffer (Beyotime, China). Lysates were sonicated for $10-15 \mathrm{~s}$ to shear DNA, heated to $100^{\circ} \mathrm{C}$ for $10 \mathrm{~min}$ and centrifuged for $10 \mathrm{~min}$ at $13,000 \times \mathrm{g}$. The protein content in the supernatant were quantified using BCA Protein Assay Kit (Beyotime, China). For protein separation, samples were prepared by mixing 5xSDS loading buffer with $10-25 \mu \mathrm{g}$ of cell lysates and separated using SDS/PAGE 10-15\% gels. Proteins were transferred to PVDF membranes, blocked with TBS-T containing 5\% milk powder for $2 \mathrm{~h}$ and then incubated with primary antibody overnight at $4{ }^{\circ} \mathrm{C}$. The post-transferred Membranes were washed for three times, followed by labeling of secondary antibodies. Signals were visualized with Odyssey ${ }^{\circledR}$ CLx Imaging System (LI-COR, USA). Due to difficulties of stripping, some plots were blotted with two different membranes but from same cell lysates.

\section{siRNA knockdown}

Cells were grown for $12 \mathrm{~h}$ to obtain $30-50 \%$ confluence. The siRNA and X-tremeGENE siRNA Transfection Reagent (Roche, USA) were separately mixed with $250 \mu \mathrm{l}$ of Opti-MEM ${ }^{\circledR}$ I Reduced Serum Medium (Gibco, USA) for $5 \mathrm{~min}$. The two mixtures were added to the cells and incubated at $37^{\circ} \mathrm{C}$ for $6 \mathrm{~h}$. Then, the serum-free medium was changed to $10 \%$ serum medium for further study. All siRNA synthesized by RiboBio (Guangzhou, China).

\section{RNA sequencing and data analysis}

Cells were lysed and total RNA was extracted as mentioned above. Samples were then submitted to Biomarker Technologies (Beijing, China), where mRNA enrichment and library preparation were performed. Samples were barcoded and run on lllumina HiSEq. Reads from RNA sequencing (RNA-seq) were mapped to Rattus norvegicus genome Rnor_6.0 (ftp://ftp.ensembl.org/pub/release-95/ fasta/rattus_norvegicus/) by using STAR (v2.5.2a) with $2 \%$ maximum mismatch. Fragments per kilobase of transcript per million fragments mapped (FPKM) and differentially expressed genes $(\mathrm{DEGs})(q$-value $<0.05)$ were generated by Cuffdiff (v2.2.1) with default parameters. The genes sensitive to Osbpl2 deficit were filtered by a minimum fold change $(\times 2)$ and a maximum false discovery rate $(\times 0.01)$ and subjected to Visualization and Integrated Discovery (DAVID) v6.8 for KEGG pathway analysis and Gene ontology (GO) analysis.

\section{Co-immunoprecipitation (Co-IP)}

HEK293Ta cells expressing the constructs of HA-ATIC and Flag-OSBPL2 were washed three times with cold PBS and then lysed with RIPA Lysis Buffer (Beyotime, China) containing complete protease inhibitors (Beyotime, China) and PhosStop (Beyotime, China) for $30 \mathrm{~min}$. Protein contents in the supernatant were incubated with Anti-FLAG ${ }^{\circledast}$ M2 Magnetic Beads (Sigma-Aldrich, Germany) overnight at $4{ }^{\circ} \mathrm{C}$. The samples were washed using RIPA Lysis Buffer (Beyotime, China) three times. Immobilized protein complexes were eluted by denaturation in $5 x$ SDS loading buffer at $100^{\circ} \mathrm{C}$ for $10 \mathrm{~min}$ and then assayed by Western blot. The protein bands were retrieved and subjected to LC-MS/MS analysis (PTM Biolabs Inc., Hanzhou, China).

\section{GST pulldown}

The proteins of His-ATIC and GST-OSBPL2 were synthesized by Zoonbio Biotechnology (Nanjing, China). Protein samples were incubated with Anti-His, Anti-GST and Protein A/G overnight at $4{ }^{\circ} \mathrm{C}$ and washed with RIPA Lysis Buffer (Beyotime, China) three times. Immobilized protein complexes were eluted and assayed by Co-IP.

\section{AICAR measurement}

AICAR was measured according to the manufacturer's instructions of AICAR ELISA Kit (Elabscience, China). Briefly, OC1 cells were washed with pre-cooled PBS and dissociated by trypsin. The cell suspension was collected, centrifuged at $1000 \times g$ for $5 \mathrm{~min}$, and suspended using pre-cooled PBS. The cells were fully lysed by repeated freeze-thaw processes. Then the samples were added to the 96 -well and incubated at $37^{\circ} \mathrm{C}$ for $90 \mathrm{~min}$. The supernatant was removed, and the remained solution was added with $100 \mu \mathrm{L}$ Biotinylated Detection Ab. The solution was aspirated or poured from each well and $350 \mu \mathrm{L}$ of wash buffer was added to each well. The samples were added with $100 \mu \mathrm{L}$ HRP conjugate, incubated $30 \mathrm{~min}$ at $37^{\circ} \mathrm{C}$ and washed $3 \times 5 \mathrm{~min}$ by PBS. Then the samples 
were added with $90 \mu \mathrm{L}$ Substrate Reagent, incubated for $15 \mathrm{~min}$ at $37^{\circ} \mathrm{C}$ and treated with $50 \mu \mathrm{L}$ of Stop Solution. The optical density (absorbance at $450 \mathrm{~nm}$ ) of the samples were determined using a micro-plate reader.

\section{Total cholesterol assay}

The cells $\left(1 \times 10^{6}\right.$ cells $)$ and tissues $(0.1 \mathrm{~g})$ were added to $1 \mathrm{ml}$ of isopropanol for homogenization in an ice bath and centrifuged at $8000 \times g$ for $10 \mathrm{~min}$ at $4{ }^{\circ} \mathrm{C}$. The supernatant was used as the total cholesterol (TC) test solution. The reaction solution $(50 \mu \mathrm{L}$ of TC standard or TC test solution) and TC working solution $(150 \mu \mathrm{L})$, were mixed in a $1 \mathrm{~mL}$ glass cuvette, kept for $24 \mathrm{~h}$ and then used to determine the absorbance $(\mathrm{A})$ at $500 \mathrm{~nm}$. TC content in tissues: $\mathrm{TC}(\mu \mathrm{mol} / \mathrm{g})=0.05 \times A_{\text {samples }} / A_{\text {standard }}$. TC content in cells: $\mathrm{TC}\left(\mu \mathrm{mol} / 10^{4}\right.$ cell $)=0.005 \times A_{\text {samples }} / A_{\text {standard }}$.

\section{ROS measurements}

The test cells or tissues were treated with or without drug and cultured for the recommended time. The cultured cells or tissues were then incubated with DCFDA (Beyotime, China) or CellROX ${ }^{\mathrm{su}}$ Deep Red Reagent (ThermoFisher, USA) at a final concentration of $5 \mu \mathrm{M}$, followed by analysis of flow cytometry. The ROS levels were quantified as the mean florescence intensity (MFI). All flow cytometry was conducted using BD FACSCalibur (Becton, Dickinson and Company, USA) and analyzed with FlowJo software (v10.5.3).

\section{Transmission electron microscopy assay}

For transmission electron microscopy (TEM) study, cells were fixed in $2 \%$ glutaraldehyde, and subjected to post-fixation with $1 \%$ osmium tetroxide in $0.1 \mathrm{M}$ cacodylate. The samples were embedded overnight with a mixture solution of $1 / 1$ ratio of propylene oxide/epoxy resin, replaced with $100 \%$ epoxy resin and then polymerized in a dry oven at $60^{\circ} \mathrm{C}$. Ultrathin sections $(70 \mathrm{~nm}$ thick) from the embedded samples were imaged by TEM (JEOL-101, Japan).

\section{Mitochondrial membrane potential assay}

JC-1 probe (Beyotime, China) was employed to measure mitochondrial depolarization in OC1 cells. Cells cultured in six-well plates for $12 \mathrm{~h}$, then incubated with JC-1 staining solution $(5 \mu \mathrm{g} / \mathrm{ml})$ at $37^{\circ} \mathrm{C}$ for $30 \mathrm{~min}$. Cells were washed three times with cold PBS. Mitochondrial membrane potential was measured by flow cytometry. Mitochondrial depolarization was indicated by an increase of the green/red fluorescence intensity ratio.

\section{Statistical analysis}

All data were presented as the mean \pm standard error of the mean (SEM). One-way ANOVA and Bonferroni's post-tests were performed to compare the differences between the groups. Other statistical significance was determined using between-group $t$-test. A $p$-values $<0.05$ was regarded as statistically significant. Statistical analysis was performed using GraphPad Prism v7 (San Diego, CA, USA).

\section{Acknowledgements}

This research was supported by the grants from the National Natural Science Foundation of China (31571302, 81771000), the Key Research and Development Program of Jiangsu Province (Social Development: BE2016762) and the Key Project of Science and Technology Innovation of Nanjing Medical University (2017NJMUCX001) to X.C.; the grants from China Postdoctoral Science Foundation (2016M600431) and Jiangsu Planned Projects for Postdoctoral Research Funds (1601071B) to J.Y.

\section{Author details}

${ }^{1}$ Department of Medical Genetics, School of Basic Medical Science, Nanjing Medical University, Nanjing, China. ${ }^{2}$ Jiangsu Key Laboratory of Xenotransplantation, Nanjing Medical University, Nanjing, China. ${ }^{3}$ The Laboratory Center for Basic Medical Sciences, Nanjing Medical University, Nanjing, China. ${ }^{4}$ Department of Otolaryngology, The First Affiliated Hospital of Nanjing Medical University, Nanjing, China

\section{Authors' contributions}

X.C. conceived the study, revised, and approved the final paper. H.W., C.L., J.Y., H.S. and C.Z. performed the experiments and analyzed the data. H.W. and J.Y. wrote the first draft of the paper. Q.W., Z.C., Y.L. and G.X. participated in the data collection and interpretation. X.C. and G.X. contributed to experiment design and conduction.

\section{Conflict of interest}

The authors declare that they have no conflict of interest.

\section{Publisher's note}

Springer Nature remains neutral with regard to jurisdictional claims in published maps and institutional affiliations.

Supplementary Information accompanies this paper at (https://doi.org/ 10.1038/s41419-019-1858-9).

Received: 11 May 2019 Revised: 25 July 2019 Accepted: 30 July 2019 Published online: 19 August 2019

\section{References}

1. Downes, C. P., Gray, A. \& Lucocq, J. M. Probing phosphoinositide functions in signaling and membrane trafficking. Trends Cell Biol. 15, 259-268 (2005).

2. Mineo, C. \& Shaul, P. W. Regulation of signal transduction by HDL. J. Lipid Res 54, 2315-2324 (2013).

3. Mouritsen, O. G. \& Zuckermann, M. J. What's so special about cholesterol? Lipids 39, 1101-1113 (2004).

4. Subczynski, W. K., Pasenkiewicz-Gierula, M., Widomska, J., Mainali, L. \& Raguz, M. High cholesterol/low cholesterol: effects in biological membranes: a review. Cell Biochem Biophys. 75, 369-385 (2017).

5. Yue, H. Y. \& Xu, J. Cholesterol regulates multiple forms of vesicle endocytosis at a mammalian central synapse. J. Neurochem 134, 247-260 (2015).

6. Bovenga, F., Sabba, C. \& Moschetta, A. Uncoupling nuclear receptor LXR and cholesterol metabolism in cancer. Cell Metab. 21, 517-526 (2015).

7. Brown, M. S., Radhakrishnan, A. \& Goldstein, J. L. Retrospective on cholesterol homeostasis: the central role of scap. Annu Rev. Biochem 87, 783-807 (2018).

8. Pramfalk, C., Eriksson, M. \& Parini, P. Cholesteryl esters and ACAT. Eur. J. Lipid Sci. Technol. 114, 624-633 (2012).

9. Sharpe, L. J. \& Brown, A. J. Controlling cholesterol synthesis beyond 3-hydroxy-3methylglutaryl-CoA reductase (HMGCR). J. Biol. Chem. 288, 18707-18715 (2013). 
10. Chistiakov, D. A., Bobryshev, Y. V. \& Orekhov, A. N. Macrophage-mediated cholesterol handling in atherosclerosis. J. Cell Mol. Med 20, 17-28 (2016).

11. Ikonen, E. Mechanisms for cellular cholesterol transport: defects and human disease. Physiol. Rev. 86, 1237-1261 (2006).

12. Puglisi, A. \& Yagci, Y. Cyclodextrin-based macromolecular systems as cholesterol-mopping therapeutic agents in niemann-pick disease type C. Macromol. Rapid Commun. 40, e1800557 (2019).

13. Xue-Shan, Z. et al. Imbalanced cholesterol metabolism in Alzheimer's disease. Clin. Chim. Acta 456, 107-114 (2016).

14. Cai, Q. et al. Effects of simvastatin on plasma lipoproteins and hearing loss in apolipoprotein E gene-deficient mice. ORL J. Otorhinolaryngol. Relat. Spec. 71, 244-250 (2009).

15. Malgrange, B., Varela-Nieto, I., de Medina, P. \& Paillasse, M. R. Targeting cholesterol homeostasis to fight hearing loss: a new perspective. Front Aging Neurosci. 7, 3 (2015)

16. Escajadillo, T., Wang, H., Li, L., Li, D. \& Sewer, M. B. Oxysterol-related-bindingprotein related Protein-2 (ORP2) regulates cortisol biosynthesis and cholesterol homeostasis. Mol. Cell Endocrinol. 427, 73-85 (2016).

17. Hynynen, R. et al. OSBP-related protein 2 is a sterol receptor on lipid droplets that regulates the metabolism of neutral lipids. J. Lipid Res 50, 1305-1315 (2009).

18. Kentala, $\mathrm{H}$. et al. Analysis of ORP2-knockout hepatocytes uncovers a novel function in actin cytoskeletal regulation. FASEB J. 32, 1281-1295 (2018).

19. Kentala, $\mathrm{H}$. et al. OSBP-related protein-2 (ORP2): a novel Akt effector that controls cellular energy metabolism. Cell Mol. Life Sci. 75, 4041-4057 (2018).

20. Koponen, A. et al. ORP2 interacts with phosphoinositides and controls the subcellular distribution of cholesterol. Biochimie 158, 90-101 (2019).

21. Xing, $\mathrm{G}$. et al. Identification of OSBPL2 as a novel candidate gene for progressive nonsyndromic hearing loss by whole-exome sequencing. Genet Med 17, 210-218 (2015).

22. Thoenes, M. et al. OSBPL2 encodes a protein of inner and outer hair cell stereocilia and is mutated in autosomal dominant hearing loss (DFNA67). Orphanet J. Rare Dis. 10, 15 (2015).

23. Kimitsuki, T. Cholesterol influences potassium currents in inner hair cells isolated from guinea pig cochlea. Auris Nasus Larynx 44, 46-51 (2017).

24. Thomas, P. V. et al. Localization and proteomic characterization of cholesterolrich membrane microdomains in the inner ear. J. Proteom. 103, 178-193 (2014).

25. Kakela, R., Tanhuanpaa, K., Laitinen, S., Somerharju, P. \& Olkkonen, V. M. Overexpression of OSBP-related protein 2 (ORP2) in $\mathrm{CHO}$ cells induces alterations of phospholipid species composition. Biochem Cell Biol. 83, 677-683 (2005).

26. Wang, $\mathrm{H}$. et al. ORP2 delivers cholesterol to the plasma membrane in exchange for phosphatidylinositol 4, 5-bisphosphate (PI(4,5)P2). Mol. Cell 73, 458-473 e457 (2019).

27. Liu, C., Yao, J., Wei, Q., Xing, G. \& Cao, X. Spatial and temporal expression patterns of Osbpl2a and Osbpl2b during zebrafish embryonic development. Int J. Pedia. Otorhinolaryngol. 84, 174-179 (2016).

28. Asby, D. J. et al. AMPK activation via modulation of de novo purine biosynthesis with an inhibitor of ATIC homodimerization. Chem. Biol. 22, 838-848 (2015).

29. Li, M. et al. Bifunctional enzyme ATIC promotes propagation of hepatocellular carcinoma by regulating AMPK-mTOR-S6 K1 signaling. Cell Commun. Signal 15, 52 (2017).

30. Liu, S. et al. AICAR-induced activation of AMPK inhibits TSH/SREBP-2/HMGCR pathway in liver. PLOS ONE 10, e0124951 (2015).

31. Tao, R., Xiong, X., DePinho, R. A., Deng, C. X. \& Dong, X. C. Hepatic SREBP-2 and cholesterol biosynthesis are regulated by FoxO3 and Sirt6. J. Lipid Res $\mathbf{5 4}$ 2745-2753 (2013).
32. Clarke, P. R. \& Hardie, D. G. Regulation of HMG-CoA reductase: identification of the site phosphorylated by the AMP-activated protein kinase in vitro and in intact rat liver. EMBO J. 9, 2439-2446 (1990).

33. Greer, E. L. et al. The energy sensor AMP-activated protein kinase directly regulates the mammalian FOXO3 transcription factor. J. Biol. Chem. 282, 30107-30119 (2007).

34. Jeon, S. M. Regulation and function of AMPK in physiology and diseases. Exp. Mol. Med 48, e245 (2016).

35. Kim, J., Kundu, M., Viollet, B. \& Guan, K. L. AMPK and mTOR regulate autophagy through direct phosphorylation of Ulk1. Nat. Cell Biol. 13, 132-141 (2011).

36. Mottillo, E. P. et al. Lack of adipocyte AMPK exacerbates insulin resistance and hepatic steatosis through brown and beige adipose tissue function. Cell Metab. 24, 118-129 (2016)

37. Viollet, B. et al. AMPK inhibition in health and disease. Crit. Rev. Biochem Mol. Biol. 45, 276-295 (2010)

38. Yavari, A. et al. Chronic activation of gamma2 AMPK induces obesity and reduces beta cell function. Cell Metab. 23, 821-836 (2016).

39. Faubert, B., Vincent, E. E., Poffenberger, M. C. \& Jones, R. G. The AMP-activated protein kinase (AMPK) and cancer: many faces of a metabolic regulator. Cancer Lett. 356, 165-170 (2015).

40. Men, Y. et al. LKB1 is required for the development and maintenance of stereocilia in inner ear hair cells in mice. PLOS ONE 10, e0135841 (2015).

41. Hill, K., Yuan, H., Wang, X. \& Sha, S. H. Noise-induced loss of hair cells and cochlear synaptopathy are mediated by the activation of AMPK. J. Neurosci. 36, 7497-7510 (2016)

42. Li, Y. et al. AMPK phosphorylates and inhibits SREBP activity to attenuate hepatic steatosis and atherosclerosis in diet-induced insulin-resistant mice. Cell Metab. 13, 376-388 (2011).

43. Greasley, S. E. et al. Crystal structure of a bifunctional transformylase and cyclohydrolase enzyme in purine biosynthesis. Nat. Struct. Biol. 8, 402-406 (2001).

44. Vergis, J. M., Bulock, K. G., Fleming, K. G. \& Beardsley, G. P. Human 5aminoimidazole-4-carboxamide ribonucleotide transformylase/inosine 5'monophosphate cyclohydrolase. A bifunctional protein requiring dimerization for transformylase activity but not for cyclohydrolase activity. J. Biol. Chem. 276, 7727-7733 (2001)

45. Liu, X. et al. Identification of ATIC as a novel target for chemoradiosensitization. Int J. Radiat. Oncol. Biol. Phys. 100, 162-173 (2018).

46. Di Berardino, F., Alpini, D., Ambrosetti, U., Amadeo, C. \& Cesarani, A. Sensorineural hearing-loss in the Smith-Lemli-Opitz syndrome. Int. J. Pediatr. Otorhinolaryngol. Extra 2, 169-172 (2007).

47. King, K. A. et al. Auditory phenotype of Niemann-Pick disease, type C1. Ear Hear 35, 110-117 (2014)

48. Kurniawan, C. et al. Gene dose of apolipoprotein $\mathrm{E}$ and age-related hearing loss. Neurobiol. Aging 33, 2230 e2237-2230 e2212 (2012).

49. Li, L., Tan, J., Miao, Y., Lei, P. \& Zhang, Q. ROS and autophagy: interactions and molecular regulatory mechanisms. Cell Mol. Neurobiol. 35, 615-621 (2015).

50. Schieber, M. \& Chandel, N. S. ROS function in redox signaling and oxidative stress. Curr. Biol. 24, R453-R462 (2014).

51. Wang, Y. et al. The STAT-ROS cycle extends IFNinduced cancer cell apoptosis. Int J. Oncol. 52, 305-313 (2018).

52. Alba, G. et al. 7-Keto-cholesterol and 25-hydroxy-1 cholesterol rapidly enhance ROS production in human neutrophils. Eur. J. Nutr. 55, 2485-2492 (2016).

53. Banfi, B. et al. NOX3, a superoxide-generating NADPH oxidase of the inner ear. J. Biol. Chem. 279, 46065-46072 (2004).

54. Du, Z. et al. A long-term high-fat diet increases oxidative stress, mitochondrial damage and apoptosis in the inner ear of D-galactose-induced aging rats. Hear Res 287, 15-24 (2012).

55. Cui, H., Kong, Y. \& Zhang, H. Oxidative stress, mitochondrial dysfunction, and aging. J. Signal Transduct. 2012, 646354 (2012). 